\title{
Index Modulation Aided Subcarrier Mapping for Dual-Hop OFDM Relaying
}

\author{
Miaowen Wen, Senior Member, IEEE, Xuan Chen, Qiang Li, Ertugrul Basar, Senior Member, IEEE, \\ Yik-Chung Wu, Senior Member, IEEE, and Wensong Zhang
}

\begin{abstract}
There is a recent surge of research interest in the study of performance-enhancing techniques for orthogonal frequency division multiplexing (OFDM)-based relay systems. Among those, subcarrier mapping has been verified to be an effective one for boosting the system capacity and improving the error performance. However, it has to be performed at the relay, which subsequently conveys the subcarrier permutation information to the destination. The existing signaling scheme occupies a portion of subcarriers to this end, leading to a loss of spectral efficiency. In this paper, we propose a novel signaling scheme to eliminate this overhead by transferring the subcarrier permutation to the mode permutation that can be implicitly conveyed without consuming additional spectrum resources. We adopt phase rotation for mode design considering both nonadaptive and adaptive modulation, and illustrate the proposed scheme by taking the dual-hop OFDM relaying with semi-blind amplify-and-forward protocol as an example. An asymptotically tight upper bound on the bit error rate (BER) of the proposed scheme is derived in closed-form over Rayleigh fading channels. BER simulation results validate the analysis and show that the proposed scheme asymptotically approaches the ideal case that assumes perfect knowledge of subcarrier permutation information at the destination and significantly outperforms the existing scheme in the asymptotic signal-to-noise ratio region at the same spectral efficiency.
\end{abstract}

Index Terms-Index modulation, multiple mode, OFDM, subcarrier permutation, dual-hop.

\section{INTRODUCTION}

A $\mathrm{S}$ a promising technology, OFDM relaying has been received considerable research attention during the past few years. Compared with single-carrier relaying, OFDM relaying brings about additional freedom for the relay to

This work was supported in part by the National Natural Science Foundation of China under Grant 61871190, in part by the Natural Science Foundation of Guangdong Province under Grant 2018B030306005, in part by the Turkish Academy of Sciences (TUBA) GEBIP Programme, in part by the State Key Laboratory of Integrated Service Networks of Xidian University under Grant ISN20-16, in part by the Pearl River Nova Program of Guangzhou under Grant 201806010171, and in part by the China Scholarship Council under Grant 201806150074

M. Wen is with the School of Electronic and Information Engineering, South China University of Technology, Guangzhou 510640, China, and also with the State Key Laboratory of Integrated Service Networks, Xidian University, Xi'an 710071, China (e-mail: eemwwen@scut.edu.cn).

X. Chen, Q. Li, and W. Zhang are with the School of Electronic and Information Engineering, South China University of Technology, Guangzhou 510640, China (e-mail: eechenxuan@mail.scut.edu.cn; eeqiangli@mail.scut.edu.cn; eez.wensong@mail.scut.edu.cn).

E. Basar is with Communications Research and Innovation Laboratory (CoreLab), Department of Electrical and Electronics Engineering, Koç University, Sariyer 34450, Istanbul, Turkey (e-mail: ebasar@ku.edu.tr).

Y.-C. Wu is with the Department of Electrical and Electronic Engineering, The University of Hong Kong, Hong Kong (e-mail:ycwu@eee.hku.hk). pair the incoming and outgoing subcarriers by their signal-tonoise ratios (SNRs), taking advantage of the diverse strength of channels associated with different hops. This so-called subcarrier/sub-channel mapping (SCM) is an important performance boosting technique for OFDM relaying with or without multiple antennas [1]-[5].

The concept of SCM was first coined in [1] assuming dualhop OFDM amplify-and-forward (AF) relaying, where SCM is verified empirically to be an effective solution for maximizing the sum SNR over all subcarrier pairs by the best-to-best (BTB) scheme: the best subcarrier of the first hop with the highest SNR is matched to the best subcarrier of the second hop, second best to second best, etc. Almost at the same time, this BTB SCM scheme was independently proved to maximize the instantaneous rate in [3] by assuming a noisefree relay. Subsequently, the same authors of [1] presented a rigorous proof for the optimality of the BTB SCM scheme in terms of both sum SNR and instantaneous rate using the majorization theory [5]. In [6], the performance gain with BTB SCM is numerically evaluated, demonstrating an average capacity increase of $10 \%-30 \%$. Moreover, numerical studies reveal that in addition to dual-hop relaying with fixed-gain or variable-gain AF protocol, BTB SCM also preserves its capacity optimality for decode-and-forward (DF) relaying [7][13], multihop relaying [13], and multi-user relaying [7], no matter with or without a direct link [11]. Even with power allocation under total or individual power constraints, the SCM problem can be separated into multiple subproblems and the BTB is still the capacity-optimal SCM scheme regardless of AF or DF relaying [9]-[11].

Attracted by the numerous benefits mentioned above, researchers have devoted great efforts to the performance analysis of the SCM schemes in terms of system capacity and bit error rate (BER) [6], [14]-[17]. While the fixed-gain AF protocol is taken into account in [6] and [15], the authors of [14] consider the variable-gain AF strategy in dual-hop relaying with BTB SCM. Surprisingly, it is found that in contrast to the capacity, applying BTB SCM cannot improve the asymptotic BER performance unless dropping several worst subcarrier pairs. A corresponding rigorous proof based on the majorization theory is given in [16], showing that as far as BER is concerned, BTB presents the best solution only in the low SNR region, while the optimal SCM scheme at medium-to-high SNR is instead the best-to-worst (BTW) matching: the best subcarrier of the first hop is paired with the worst subcarrier of the second hop, second best with second worst, and so on. Further, the authors of [17] perform the exact 
BER analysis for both BTB and BTW SCM schemes assuming a fixed-gain AF relaying, and concluded that the relay system may switch from BTB to BTW to achieve optimum BER performance.

The concept of BTB or BTW SCM is simple yet effective. However, it requires the relay to calculate the subcarrier reordering function based on the local channel state information (CSI) and then transmits it to the destination. Although this practical issue has been highlighted in many studies such as [3] and [17], to the best of our knowledge, only [3] partially addresses it by resorting to chunk-based mapping, which first groups adjacent subcarriers in chunks and then performs chunk permutation according to the average chunk's SNRs. Indeed, the chunk-based mapping is much more efficient than the original subcarrier-to-subcarrier mapping when the number of subcarriers is large. However, it may not be applied to the scenarios with a small number of subcarriers/subchannels, such as multi-input-multi-output relaying, multi-user OFDM relaying, and their combination. Moreover, the existing method for signaling the chunk permutation information [3] still relies on explicit transmissions by occupying a portion of subcarriers, whose required amount scales up quickly with the number of chunks, resulting in non-negligible signaling overhead especially for low data rate services. ${ }^{1}$ In view of lacking a low-cost signaling method in the literature, the goal of this paper is to solve this problem.

Our solution is inspired by the recently proposed multiplemode OFDM with index modulation (MM-OFDM-IM) technique [18]-[20], which is a variant of the OFDM-IM technique [21]-[23]. Here, IM refers to a novel modulation concept that uses the index of some building block of a communication system to convey information [24]-[27], and OFDM-IM is a realization of IM in frequency domain that adopts the indices of active OFDM subcarriers for IM purposes. Noticing the $\mathrm{p}$ resence of idle subcarriers limits the spectral efficiency (SE) of OFDM-IM systems, MM-OFDM-IM activates all subcarriers to transmit multiple distinguishable signal constellations (or modes, alternatively) to improve the SE. Particularly, MMOFDM-IM encodes the IM bits onto the full permutation of modes, leading to a faster increase of the number of IM bits than OFDM-IM. Design guidelines and low-complexity subcarrier-wise detection algorithms for MM-OFDM-IM are proposed in [18] and [20], based on which MM-OFDM-IM is shown to outperform nearly all existing OFDM-IM related techniques with a similar receiver complexity. Recalling that the subcarrier/chunk reordering function can be expressed as a full permutation of subcarriers/chunks, we aim to seek a possible solution to the signaling overhead problem by building a relationship with MM-OFDM-IM. However, it can be very challenging due to the following two aspects. First, in MM-OFDM-IM all transmitted symbols are drawn

\footnotetext{
${ }^{1}$ In [3], it is suggested to transmit the index of each paired chunk. However, a more efficient way to save signaling bits could be that the relay takes the chunk permutation as a whole for bit encoding. For example, assuming four chunks encoding the index of its paired chunk for each chunk requires 2 bits, amounting to 8 bits for signaling the reordering function. However, encoding the chunk permutation needs only $\left\lceil\log _{2}(4 !)\right\rceil=5$ bits to this end. Therefore, for a fair comparison we adopt the latter method instead of the former one as a benchmark in this paper.
}

from predesigned modes and a mode permutation is selected according to the incoming bits of the transmitter, whereas the reordering function is generated by the local CSI at the relay and all modes have to be created from the signal received by the relay adaptively. Second, in MM-OFDM-IM all modes are fixed even though the cardinality of one mode is allowed to be different from that of any other mode [20], whereas unfixed modes are necessary for fully conveying the reordering information in adaptive modulation scenarios, where a better subcarrier/chunk pair always employs a signal constellation of a larger cardinality and vice versa.

Against this background, the contributions of this paper are summarized as follows:

- We propose an IM-aided SCM (SCM-IM) scheme to eliminate the signaling overhead by taking the dual-hop OFDM AF relaying with all nodes configured with a single antenna for illustration purposes, though its principle is general and applicable to multiple-antenna/hop setting. In the proposed scheme, the relay adaptively creates multiple modes via phase rotation and transfers the subcarrier/chunk permutation information to the mode permutation, such that the reordering information is implicitly transmitted to the destination without occupying additional resources. All modes after phase rotation do not overlap, making the proposed scheme support both adaptive and non-adaptive modulation.

- We present a new framework for the analysis of the upper bound on the BER of the proposed scheme applicable to both adaptive and non-adaptive modulation, which is completely different from the existing analytical method$\mathrm{s}$ in the literature. The proposed analytical framework isolates the error events that the mode permutation is detected incorrectly and correctly, and qualifies their respective contributions to the BER, providing valuable insight into the nature of the proposed scheme.

- We verify the accuracy of our analysis and compare the BER performance of the proposed scheme with that of the existing one in [3] via computer simulations for both non-adaptive and adaptive modulation taking into account both BTB and BTW. The proposed scheme is shown to significantly outperform the existing scheme in [3] and asymptotically approach the ideal case that assumes perfect knowledge of subcarrier permutation information at the destination in the asymptotic SNR region at the same SE.

The remainder of this paper is organized as follows. Section II describes the system model of OFDM AF relaying and the concept of SCM. Section III gives the implementation of SCM, including the conventional scheme and the proposed SCM-IM scheme. The error performance of SCM-IM is analyzed in Section IV, followed by the computer simulation results in Section V. Finally, Section VI concludes the paper.

Notation: Column vectors and matrices are denoted by lowercase and capital bold letters, respectively. Superscript $T$ stands for transposition. $\operatorname{diag}(\cdot)$ transforms a vector into a diagonal matrix. $\mathcal{C} \mathcal{N}\left(0, \sigma^{2}\right)$ represents the complex Gaussian distribution with zero mean and variance $\sigma^{2}$. The probability 
of an event and the probability density function (PDF) are denoted by $\operatorname{Pr}(\cdot)$ and $f(\cdot)$, respectively. $E\{\cdot\}$ denotes the expectation operation. $Q(\cdot), K_{0}(\cdot)$, and $K_{1}(\cdot)$ are the Gaussian $Q$-function, zero and first order modified Bessel functions of the second kind, respectively. $\operatorname{Ei}(\cdot)$ and $\mathcal{U}(\cdot)$ denote the exponential integral function and the unit-step function, respectively. $C(\cdot, \cdot)$ and $\lceil\cdot\rceil$ are the binomial coefficient and the ceil function, respectively.

\section{SySTEM MODEL AND SCM INTRODUCTION}

We consider a dual-hop OFDM AF relay system of $N$ subcarriers, in which a base station communicates with a mobile station with the aid of an AF relay. All devices are equipped with a single transmit/receive antenna. It is assumed that perfect time-frequency synchronization can be achieved. Without loss of generality, downlink transmission is taken as an illustrative example in the sequel unless otherwise specified. The complete communication consists of two time slots.

\section{A. Broadcasting Phase}

In the first time slot, the base station transmits OFDM signals to the relay. The frequency-domain received signal at the relay is given by

$$
Y_{R}(i)=\sqrt{\rho_{B}} H_{1}(i) X_{i}+W_{1}(i), \quad i=1, \ldots, N,
$$

where $H_{1}(i), X_{i} \in \mathcal{S}_{i}$, and $W_{1}(i)$, respectively, are the channel frequency response of the first hop, the transmitted symbol, and the additive white Gaussian noise (AWGN) sample at the relay on the $i$-th subcarrier, $\mathcal{S}_{i}$ is the normalized $M_{i}$-ary PSK/quadrature amplitude modulation (QAM) constellation, and $\rho_{B}$ denotes the transmit power of the base station. It is assumed that $H_{1}(i)$ and $W_{1}(i)$ follow the distributions $\mathcal{C N}\left(0, \Omega_{1}\right)$ and $\mathcal{C N}\left(0, N_{01}\right)$, respectively. Notice that $S_{i}$ accommodates both non-adaptive modulation and adaptive modulation. For non-adaptive modulation, we have $M_{1}=\cdots=M_{N}=M$, while for adaptive modulation, $M_{i}, i=1, \ldots, N$, are not necessarily equal. ${ }^{2}$

\section{B. SCM Assisted Relaying}

Provided that the CSI of both hops are available at the relay, the relay calculates the subcarrier reordering function $\mathcal{V}: v(i) \rightarrow i$, where $i, v(i) \in\{1, \ldots, N\}$. By such, the $v(i)$ th subcarrier of the first hop is coupled with the $i$-th subcarrier of the second hop, according to the concept of BTB or BTW SCM. It is obvious that there are $N$ ! different coupling patterns in total, including the special case of relaying without SCM, i.e., $v(i)=i$. After performing subcarrier reordering on $\mathbf{y}_{R}=\left[Y_{R}(1), \ldots, Y_{R}(N)\right]^{T}$ with $\mathcal{V}$, the relay forwards the reordered signal, i.e., $\tilde{\mathbf{y}}_{R}=\left[Y_{R}(v(1)), \ldots, Y_{R}(v(N))\right]^{T}$, to the mobile station with a fixed gain of $G$, where $Y_{R}(i)$ with $i \in\{1, \ldots, N\}$ is the received signal on the $i$-th subcarrier

\footnotetext{
${ }^{2}$ Note that the adaptive modulation considered in this paper belongs to the family of variable-rate transmission [30], [31], which enjoys simple implementation.
}

at the relay. The received signal at the mobile station in the frequency domain can be expressed as

$$
\begin{aligned}
Y_{M}(i) & =G H_{2}(i) Y_{R}(v(i))+W_{2}(i) \\
& =G H_{2}(i) H_{1}(v(i)) X_{v(i)}+G H_{2}(i) W_{1}(v(i))+W_{2}(i),
\end{aligned}
$$

where $H_{2}(i)$ and $W_{2}(i)$ are the channel frequency response of the second hop and the AWGN sample at the mobile station on the $i$-th subcarrier, which follow the distributions $\mathcal{C N}\left(0, \Omega_{2}\right)$ and $\mathcal{C N}\left(0, N_{02}\right)$, respectively.

In this paper, we consider the fixed-gain operation at the relay assuming knowledge of the statistical CSI of the first hop [28]. In this semi-blind scenario, the fixed gain can be calculated by [29]

$$
\begin{aligned}
G^{2} & =E\left\{\frac{\rho_{R}}{\rho_{B}\left|H_{1}(i)\right|^{2}+N_{01}}\right\} \\
& =-\frac{\rho_{R}}{\rho_{B} \Omega_{1}} \exp \left(\frac{1}{\bar{\gamma}_{1}}\right) \operatorname{Ei}\left(-\frac{1}{\bar{\gamma}_{1}}\right),
\end{aligned}
$$

and the instantaneous end-to-end SNR on the $i$-th subcarrier can be expressed as

$$
\gamma_{i}=\frac{\frac{\rho_{B}\left|H_{1}(v(i))\right|^{2}}{N_{01}} \cdot \frac{\rho_{R}\left|H_{2}(i)\right|^{2}}{N_{02}}}{\frac{\rho_{R}\left|H_{2}(i)\right|^{2}}{N_{02}}+\frac{\rho_{R}}{G^{2} N_{01}}}=\frac{\gamma_{1, v(i)} \gamma_{2, i}}{\gamma_{2, i}+C}
$$

where $\rho_{R}$ represents the transmit power of the relay, $C=$ $\rho_{R} /\left(G^{2} N_{01}\right), \quad \bar{\gamma}_{1}=\rho_{B} \Omega_{1} / N_{01}$ is the average SNR for the first hop, $\gamma_{1, v(i)}=\rho_{B}\left|H_{1}(v(i))\right|^{2} / N_{01}$ is the instantaneous SNR on the $v(i)$-th subcarrier of the first hop, and $\gamma_{2, i}=\rho_{R}\left|H_{2}(i)\right|^{2} / N_{02}$ is the instantaneous SNR on the $i$ th subcarrier of the second hop. Note that (3) is valid under the assumption that all underlying random variables follow complex Gaussian distribution.

After obtaining $H_{2}(i) H_{1}(v(i))$ through channel estimation, $X_{v(i)}$ can be estimated at the mobile station as

$$
\hat{X}_{v(i)}=\underset{X_{v(i)}}{\arg \min }\left|Y_{M}(i)-\sqrt{G} H_{2}(i) H_{1}(v(i)) X_{v(i)}\right|^{2},
$$

where $\hat{X}_{v(i)}$ is the estimate of $X_{v(i)}$, and $i=1, \ldots, N$. However, since the order is scrambled, the knowledge of $\mathcal{V}$ is required at the mobile station to recover $X_{i}$ from $X_{v(i)}$. Therefore, the transmission of $\mathcal{V}$ to the mobile station plays a vital role in practical SCM systems.

\section{PRACTICAl Implementation OF SCM}

As stated in Section II, how to signal the reordering function to the mobile station is a key issue for the practical implementation of SCM. However, the subcarrier-based SCM in Section II.B involves a high computational burden due to the calculation of $\mathcal{V}$ and the subcarrier reordering of $N$ subcarriers, where $N$ is often a large number. Hence, SCM is usually performed in the chunk-based manner for an efficient SCM [3]. Specifically, $N$ subcarriers are first grouped into $n$ chunks, each of which includes $u=N / n$ subcarriers. As suggested in [6] and [15], the $n$ chunks should be drawn in an interleaved manner to benefit the diversity gain from the independent fading. We assume as in [3], [6], and [15] 

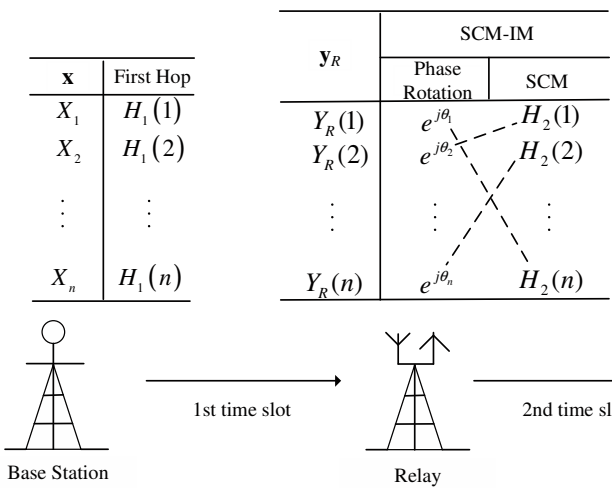

st time slot
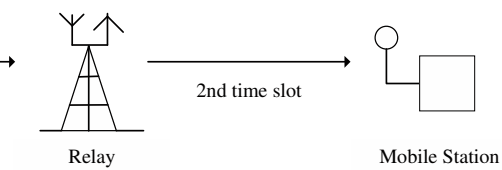

Fig. 1. Schematic diagram of OFDM AF relaying with SCM-IM.

that each chunk has a bandwidth smaller than the coherence bandwidth such that all the subcarriers it includes experience nearly frequency non-selective fading. Therefore, the relay can simply set up the chunk reordering function, $\mathcal{V}: v(k) \rightarrow k$, according to the average chunk's SNRs of both hops, where $k, v(k) \in\{1, \ldots, n\}$. Based on $\mathcal{V}$, the $v(k)$-th chunk of the first hop would be mapped to the $k$-th chunk of the second hop. By rewriting $\mathbf{y}_{R}$ as $\mathbf{y}_{R}=\left[\left(\mathbf{y}_{R}^{1}\right)^{T}, \ldots,\left(\mathbf{y}_{R}^{n}\right)^{T}\right]^{T}$, where $\mathbf{y}_{R}^{k}=\left[Y_{R}((k-1) u+1), \ldots, Y_{R}(k u)\right]^{T}$ is the $k$-th chunk of $\mathbf{y}_{R}$, the reordered signal can be given by $\tilde{\mathbf{y}}_{R}=$ $\left[\left(\mathbf{y}_{R}^{v(1)}\right)^{T}, \ldots,\left(\mathbf{y}_{R}^{v(n)}\right)^{T}\right]^{T}$. Based on this chunk-based SCM, in this section, we first review the conventional scheme, and then we propose the SCM-IM scheme, for signaling the reordering function from the relay to the mobile station. Without loss of generality, we take the case of $u=1$, i.e., $n=N$, as an illustrative example in the sequel.

\section{A. Conventional Scheme}

In conventional SCM scheme [3], extra bits encoded into modulated symbols are transmitted to the mobile station for signaling the reordering function. Since there are $n$ ! permutations in total, $\left\lceil\log _{2}(n !)\right\rceil$ bits are needed to encode the chunk permutation. These $\left\lceil\log _{2}(n !)\right\rceil$ bits are modulated into PSK/QAM symbols and then transmitted without SCM along with $\tilde{\mathbf{y}}_{R}$ to the mobile station. At the receiver side, the $\left\lceil\log _{2}(n !)\right\rceil$ bits and $X_{v(k)}$ for $k=1, \ldots, n$, are detected independently, from which $\mathbf{x}=\left[X_{1}, \ldots, X_{n}\right]^{T}$ is recovered.

It is clear that the $\left\lceil\log _{2}(n !)\right\rceil$ overhead bits should occupy extra subcarriers, thus inevitably degrading the system SE. To solve this problem, in the next subsection, we will propose SCM-IM scheme by embedding the chunk permutation information into the information-bearing signal itself.

\section{B. Proposed SCM-IM}

Motivated by MM-OFDM-IM, we propose to encode those extra bits into the full permutation of multiple distinguishable signal constellations such that the subcarrier reordering function $\mathcal{V}$ can be transmitted implicitly along with the information-bearing signal to the mobile station, saving the signaling overhead.
1) Principle Description: The schematic diagram of SCM-IM is depicted in Fig. 1. In SCM-IM with PSK signaling, the $k$-th chunk (subcarrier) of the received signal, namely $Y_{R}(k)$, is first rotated with angle $\theta_{k}$, forming $\mathbf{y}_{R}^{\prime}=\left[Y_{R}(1) e^{j \theta_{1}}, \ldots, Y_{R}(n) e^{j \theta_{n}}\right]^{T}$, and then $\mathbf{y}_{R}^{\prime}$ is reordered according to $\mathcal{V}$, which generates $\tilde{\mathbf{y}}_{R}^{\prime}=\left[Y_{R}(v(1)) e^{j \theta_{v(1)}}, \ldots, Y_{R}(v(n)) e^{j \theta_{v(n)}}\right]^{T}$, where $k \in$ $\{1, \ldots, n\}$ and $\left\{\theta_{1}, \ldots, \theta_{n}\right\}$ are $n$ different angles to be optimized. ${ }^{3}$ After this point, $\tilde{\mathbf{y}}_{R}{ }_{R}$ is forwarded to the mobile station over the channel $\mathbf{h}_{2}=\left[H_{2}(1), \ldots, H_{2}(n)\right]^{T}$. As a result, the frequency-domain received signal at the mobile station for the $k$-th chunk can be expressed as

$$
\begin{aligned}
Y_{M}(k)= & G H_{2}(k) Y_{R}(v(k)) e^{j \theta_{v(k)}}+W_{2}(k) \\
= & G H_{2}(k) H_{1}(v(k)) X_{v(k)} e^{j \theta_{v(k)}}+W_{2}(k) \\
& +G H_{2}(k) W_{1}(v(k)) e^{j \theta_{v(k)}}, \quad k=1, \ldots, n .
\end{aligned}
$$

We observe from (6) that symbols in different chunks are rotated with different angles. Hence, the chunk permutation information is transferred to the mode permutation by $X_{v(k)} e^{j \theta_{v(k)}, k}=1, \ldots, n$, which are drawn from $n$ distinguishable constellations. Therefore, from (6), we have the optimal maximum-likelihood (ML) detection, i.e.,

$\left(\hat{\mathbf{x}}^{\prime}, \hat{\mathbf{r}}\right)=\underset{\mathbf{x}^{\prime}, \mathbf{r}}{\arg \min } \sum_{k=1}^{n} \mid Y_{M}(k)-G H_{2}(k) H_{1}(v(k)) X_{v(k)} e^{\left.j \theta_{v(k)}\right|^{2},(7)}$

where $\mathbf{x}^{\prime}=\left[X_{v(1)}, \ldots, X_{v(n)}\right]^{T}, \mathbf{r}=\left[e^{j \theta_{v(1)}}, \ldots, e^{j \theta_{v(n)}}\right]^{T}$, and $H_{2}(k) H_{1}(v(k))$ can be derived through channel estimation; $\hat{\mathbf{x}}^{\prime}$ and $\hat{\mathbf{r}}$ are the estimates of $\mathbf{x}^{\prime}$ and $\mathbf{r}$, respectively. Finally, $\mathbf{x}$ can be reconstructed from $\hat{\mathbf{x}}^{\prime}$ using $\hat{\mathbf{r}}$. The computational complexity of the optimal ML detector in (7) in terms of complex multiplications is of order $\mathcal{O}\left(n ! M^{n}\right)$, which becomes burdensome for large values of $n$ and $M$. Fortunately, the subcarrier-wise detector proposed in [18] can be employed to reduce the computational complexity. With the subcarrier-wise detector, the modes carried on the $n$ chunks are determined by following the decreasing order of the average chunk's SNRs. The subcarrier-wise detector achieves nearoptimal error performance with greatly reduced computational complexity of order $\mathcal{O}\left(M n^{2} / 2+M n / 2\right) .{ }^{4}$ Different from the conventional SCM scheme that employs extra bits to convey the reordering information, SCM-IM eliminates the signaling overhead completely.

The values of $\left\{\theta_{1}, \ldots, \theta_{n}\right\}$ should be carefully designed such that the resulting $n$ signal constellations are as distinguishable as possible for achieving good error performance. For non-adaptive modulation, we select $\left\{\theta_{1}, \ldots, \theta_{n}\right\}$ according to the mode selections proposed in [18], while for adaptive modulation, the selection of $\left\{\theta_{1}, \ldots, \theta_{n}\right\}$ is different from that in [20].

\footnotetext{
${ }^{3}$ Note that SCM-IM can also use QAM signaling. However, instead of phase rotation as in the PSK scenario, linear transform should be performed on $\mathbf{y}_{R}$ at the relay to generate multiple modes as described in [32]. Since the remaining operations are similar to those for the PSK scenario, we focus on PSK signaling in this paper.

${ }^{4}$ Note that the subcarrier-wise detector is applicable to the case when $u>1$. In this case, the computational complexity in terms of complex multiplications is of order $\mathcal{O}\left(\right.$ Mun $^{2} / 2+$ Mun $\left./ 2\right)$.
} 


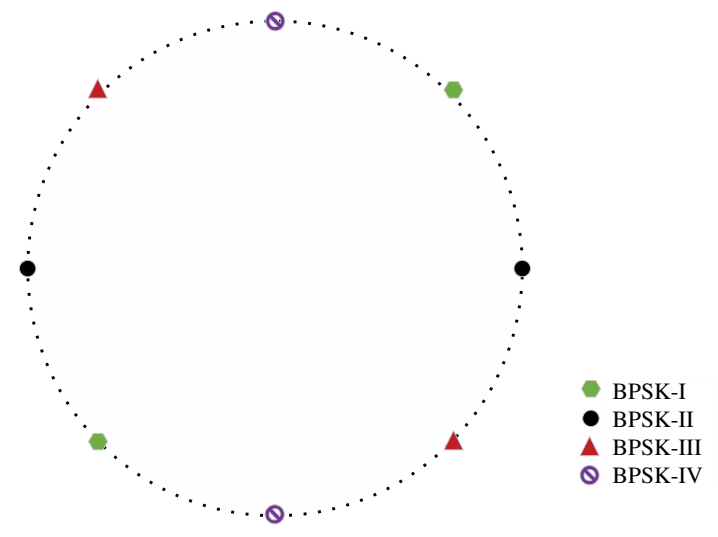

Fig. 2. Four optimal modes from the 8-PSK constellation without adaptive modulation $(n=4$ and $M=2)$.

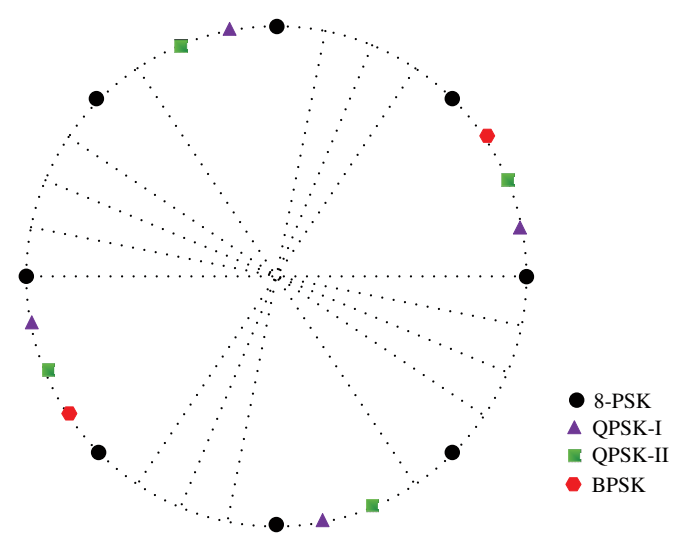

Fig. 3. Four optimal modes from the 32-PSK constellation with the adaptive modulation $\left(M_{1}=8, M_{2}=M_{3}=4\right.$, and $\left.M_{4}=2\right)$.

- Non-Adaptive Modulation: In this case, the optimal $n$ modes are shown to be extracted from the regular $n M$ PSK constellation [18]. Therefore, we choose $\theta_{k}=$ $2 \pi(k-1) /(n M), k=1, \ldots, n$. Fig. 2 presents an example of the resulting $n$ modes for $n=4$ and $M=2$.

- Adaptive Modulation: In this case, all signal points from $n$ modes are drawn from the regular normalized $n M_{m^{-}}$ ary PSK constellation, where $M_{m}=\max \left\{M_{1}, \ldots, M_{n}\right\}$, and we choose $\theta_{k}=2 \pi(k-1) /\left(n M_{m}\right), k=1, \ldots, n$. By this design, the mode of $M_{k}$ constellation points is visualized as an $M_{k}$-ary PSK constellation with $M_{k}$ practical constellation points, where $k=1, \ldots, n$, and we can avoid overlapping between any two modes after phase rotation. An example of mode selection for adaptive modulation with $M_{1}=8, M_{2}=M_{3}=4$, and $M_{4}=2$, is shown in Fig. 3, where $\theta_{1}=0, \theta_{2}=\pi / 16, \theta_{3}=\pi / 8$, and $\theta_{4}=3 \pi / 16$.

2) Initialization Protocol: To perform SCM-IM, the CSI of the base station $\rightarrow$ relay and relay $\rightarrow$ mobile station channels are required at the relay for the calculation of reordering function. This can be accomplished through the following 3-time slot initialization protocol with the assumption that the channels do not change significantly between uplink and downlink transmission, as shown in Fig. 4. During the first time slot, the base station transmits one OFDM packet to the
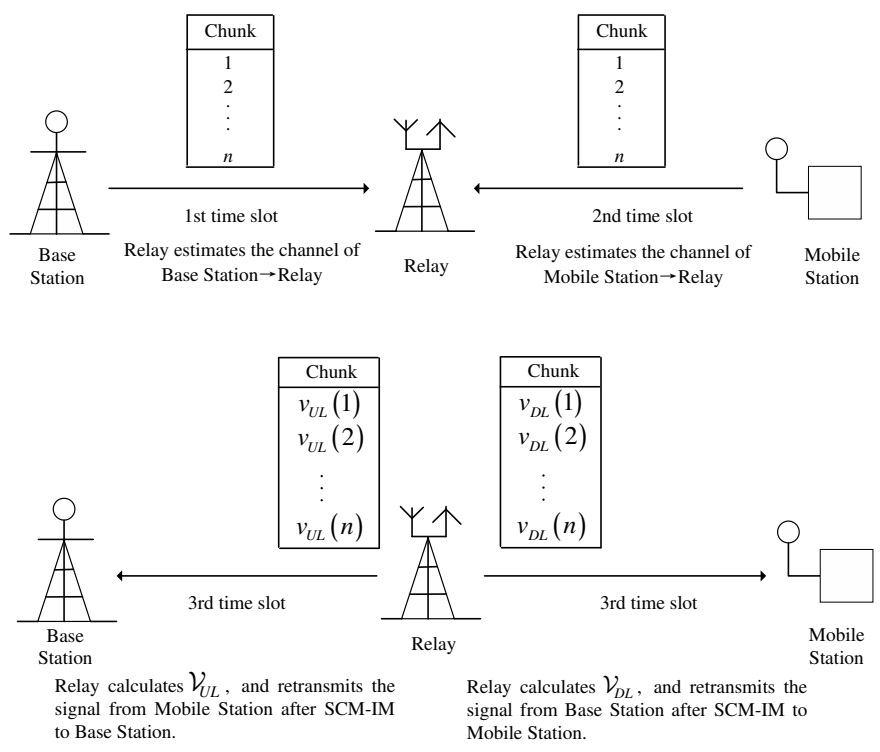

Fig. 4. Protocol overview for initialization.

relay with non-adaptive modulation since the base station has no CSI at this stage, and then the relay estimates the base station $\rightarrow$ relay channel by utilizing the pilot bits. Similarly, in the second time slot, the mobile station employs non-adaptive modulation for the transmission of one OFDM packet to the relay, and then the relay uses the pilot bits to estimate the relay $\rightarrow$ mobile station channel. After obtaining the CSI of both hops, the relay calculates the subcarrier reordering functions, namely $\mathcal{V}_{D L}$ and $\mathcal{V}_{U L}$, for downlink and uplink, respectively, according to the rule of BTB or BTW SCM. During the third time slot, the relay performs SCM-IM on the received signal from the base station (mobile station) based on $\mathcal{V}_{D L}\left(\mathcal{V}_{U L}\right)$, and then retransmits the phase rotated and reordered signals to the mobile station (base station). The mobile station (base station) receives the OFDM packet and estimates the overall base station $\rightarrow$ mobile station channel, thus reconstructing the original OFDM symbol from the base station (mobile station). After initialization, both the base station and mobile station obtain the CSI of the base station $\rightarrow$ mobile station link, which means they can (but not necessarily have to) apply the adaptive modulation in the following transmissions.

\section{Performance Analysis}

\section{A. Non-Adaptive Modulation}

In this subsection, we derive a tight upper bound on the BER of the OFDM AF relaying with SCM-IM, assuming nonadaptive modulation and the optimal ML detection.

Obviously, the bit errors can be categorized into two cases, depending on whether the mode permutation is detected correctly. We first provide two lemmas to characterize the bit error probabilities (BEPs) for these two cases, and then obtain the overall BER upper bound.

Lemma 1: For both BTB and BTW SCM schemes, the BEP contributed by the error event that the mode permutation is 
detected incorrectly is given by

$$
\begin{aligned}
P_{b 1} \approx & \frac{1}{p n !} \sum_{i=1}^{n !} \sum_{j=1, j \neq i}^{n !} \operatorname{Pr}\left(I_{i} \rightarrow I_{j}\right)\left[D\left(I_{i}, I_{j}\right) P_{b-S C M}(M) \log _{2}(M)\right. \\
& \left.+\bar{D}\left(I_{i}, I_{j}\right)\left(1-(1 / 2)^{\log _{2}(M)}\right) \log _{2}(M)\right],
\end{aligned}
$$

where $D\left(I_{i}, I_{j}\right)$ and $\bar{D}\left(I_{i}, I_{j}\right)$, respectively, denote the numbers of subcarriers whose modes are detected correctly and incorrectly, $p=n \log _{2}(M), P_{b-S C M}(M)$ that will be provided in Lemma 2 is the BEP of OFDM AF relay systems with perfect (error-free) SCM detection, $I_{i}$ denotes the $i$-th permutation, and $\operatorname{Pr}\left(I_{i} \rightarrow I_{j}\right)$ defines the error probability of detecting $I_{i}$ as $I_{j}$ that can be derived as

$$
\begin{aligned}
\operatorname{Pr}\left(I_{i} \rightarrow I_{j}\right) \approx & \left(1-P_{b-S C M}(n M) \log _{2}(n M)\right)^{D\left(I_{i}, I_{j}\right)} \\
& \times\left(P_{b-S C M}(n M) \log _{2}(n M)\right)^{\bar{D}\left(I_{i}, I_{j}\right)} .
\end{aligned}
$$

Proof: The proof is based on the union bounding technique by only counting the error events in which the mode permutation is detected incorrectly. For each of those error events, we calculate the BEPs for subcarriers whose carrying modes are detected correctly and incorrectly, respectively. Please see Appendix A for more details.

Lemma 2: When the mode permutation is detected perfectly, the BEP of the OFDM AF relaying with SCM-IM can be expressed as

$$
\begin{aligned}
P_{b-S C M}(M) & =\frac{1}{n} \sum_{k=1}^{n} P_{b-S C M}(M, k), \\
P_{b-S C M}(M, k) & =\frac{A}{12} \mathcal{M}_{\gamma_{k}}\left(\frac{B}{2}\right)+\frac{A}{4} \mathcal{M}_{\gamma_{k}}\left(\frac{2 B}{3}\right),
\end{aligned}
$$

where $A=1$ for $M=2$, while $A=2 / \log _{2}(M)$ for $M \geq 4$, $B=2 \sin ^{2}(\pi / M)$, and $\mathcal{M}_{\gamma_{k}}(\cdot)$ is the moment generating function (MGF) of $\gamma_{k}$, which is given by

$$
\begin{aligned}
\mathcal{M}_{\gamma_{k}}^{B T B}(s)= & \frac{1}{\bar{\gamma}_{1}} \sum_{j=0}^{k-1} \sum_{i=0}^{k-1} \frac{a_{j} a_{i}}{V_{j}(s)}\left[\frac{1}{b_{i}}-\exp \left(\frac{C A_{j, i}}{V_{j}(s)}\right)\right. \\
& \left.\times \operatorname{Ei}\left(-\frac{C A_{j, i}}{V_{j}(s)}\right)\left(\frac{C}{\bar{\gamma}_{2}}-\frac{C A_{j, i}}{b_{i} V_{j}(s)}\right)\right],
\end{aligned}
$$

for BTB SCM, and

$$
\begin{aligned}
\mathcal{M}_{\gamma_{k}}^{B T W}(s)= & \frac{1}{\bar{\gamma}_{1}} \sum_{j=0}^{k-1} \sum_{i=0}^{n-k} \frac{a_{j} \delta_{i}}{V_{j}(s)}\left[\frac{1}{\varsigma_{i}}-\exp \left(\frac{C B_{j, i}}{V_{j}(s)}\right)\right. \\
& \left.\times \operatorname{Ei}\left(-\frac{C B_{j, i}}{V_{j}(s)}\right)\left(\frac{C}{\bar{\gamma}_{2}}-\frac{C B_{j, i}}{\varsigma_{i} V_{j}(s)}\right)\right],
\end{aligned}
$$

for BTW SCM with

$$
\begin{gathered}
a_{i}=(-1)^{i} n C(n-1, k-1) C(k-1, i), \quad b_{i}=i+n-k+1, \\
\delta_{i}=(-1)^{i} n C(n-1, k-1) C(n-k, i), \quad \varsigma_{i}=i+k, \\
A_{j, i}=b_{j} b_{i} / \bar{\gamma}_{1} \bar{\gamma}_{2}, \quad B_{j, i}=b_{j} \varsigma_{i} / \bar{\gamma}_{1} \bar{\gamma}_{2},
\end{gathered}
$$

and

$$
V_{j}(s)=s+b_{j} / \bar{\gamma}_{1}
$$

Proof: In this case, the BEP analysis is similar to that of dual-hop OFDM systems with ideal SCM. Please refer to Appendix B for more details.

Based on Lemma 1 and Lemma 2, we have the following proposition.

Proposition: An upper bound on the BER of OFDM AF relaying with BTB or BTW SCM-IM is given by

$$
P_{b} \leq P_{b 1}+P_{b-S C M}(M) .
$$

Proof: See Appendix C.

We observe from (47) in Appendix $C$ that the diversity order of OFDM AF relaying with BTB or BTW SCM-IM is the smaller one between the two diversity orders captured by $P_{b 1}$ and $P_{b-S C M}(M)$. It is clear that $P_{b-S C M}(M)$ shows a diversity order of unity since SCM does not change the diversity order of classical OFDM. The diversity order for $P_{b 1}$ is determined by $\bar{D}\left(I_{i}, I_{j}\right)$ in (9). Since the minimum value of $\bar{D}\left(I_{i}, I_{j}\right)$ is $2, P_{b 1}$ exhibits a diversity order of two. Therefore, OFDM AF relaying with SCM-IM achieves a diversity order of unity.

\section{B. Adaptive Modulation}

In this subsection, we derive a BER upper bound of the OFDM relaying with SCM-IM considering adaptive modulation. In the adaptive scenario, multiple constellation orders should be taken into account in the derivation of the BER upper bound.

Lemma 3: For the adaptive scenario, the probability of the event $I_{i} \rightarrow I_{j}$ is upper bounded by

$$
\begin{aligned}
\operatorname{Pr}\left(I_{i} \rightarrow I_{j}\right) \leq & \left(1-P_{b-S C M}\left(n M_{m}\right) \log _{2}\left(n M_{m}\right)\right)^{|\mathbf{U}|} \\
& \times\left(P_{b-S C M}\left(n M_{m}\right) \log _{2}\left(n M_{m}\right)\right)^{|\overline{\mathbf{U}}|} \\
= & P_{U}
\end{aligned}
$$

and the BEP contributed by the error event that the mode permutation is detected incorrectly is upper bounded by

$$
\begin{aligned}
P_{b 1} \leq & \frac{1}{\sum_{k=1}^{n} \log _{2}\left(M_{k}\right) n !} \sum_{i=1}^{n !} \sum_{j=1, j \neq i}^{n !} P_{U} \\
& \times\left[\sum_{l=1}^{|\mathbf{U}|} \mathrm{P}_{b-S C M}\left(M_{I_{i, \mathbf{U}(l)}}\right) \log _{2}\left(M_{I_{i, \mathbf{U}(l)}}\right)\right. \\
& +\sum_{k=1}^{|\overline{\mathbf{U}}|}\left(1-2^{\left.\left.-M_{I_{i, \overline{\mathbf{U}}(k)}}\right) \log _{2}\left(M_{I_{i, \overline{\mathbf{U}}(k)}}\right)\right],}\right.
\end{aligned}
$$

where $\mathbf{U}$ and $\overline{\mathbf{U}}$ are two sets of cardinalities $|\mathbf{U}|$ and $|\overline{\mathbf{U}}|$ with $|\mathbf{U}|+|\overline{\mathbf{U}}|=n$, respectively, containing the indices of subcarriers, whose carrying modes are detected correctly and incorrectly, respectively, and $I_{i, j}$ denotes the $j$-th mode associated with the $i$-th mode permutation, with $i \in\left\{1, \ldots, 2^{n}\right\}$ and $j \in\{1, \ldots, n\}$.

Proof: See Appendix D.

On the other hand, the BEP contributed by the error event that the mode permutation is detected correctly can be modified as

$$
P_{b-S C M}=\frac{1}{p} \sum_{k=1}^{n} P_{b-S C M}\left(M_{k}\right) \log _{2}\left(M_{k}\right) .
$$




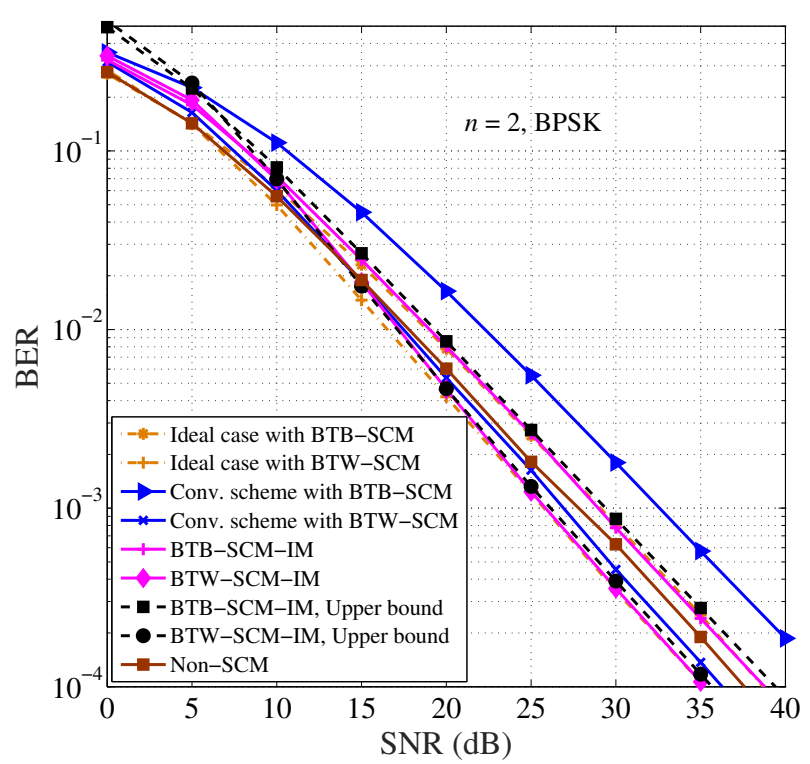

Fig. 5. Performance comparison among different (non-) SCM schemes with $n=2$ and BPSK considering both BTB and BTW.

Finally, a BER upper bound can be obtained by substituting (20) and (21) into (18).

\section{Simulation Results And Discussion}

In this section, we perform Monte Carlo simulations to evaluate the uncoded BER performance of the proposed SCMIM scheme over Rayleigh fading channels. In the simulation$\mathrm{s}$, a balanced dual-hop relaying topology with $\rho_{B}=\rho_{R}$, $N_{01}=N_{02}$, and $\bar{\gamma}_{1}=\bar{\gamma}_{2}$ is considered. We follow the chunk-based SCM idea in [3]. Since the chunk-based OFDM system becomes equivalent to a multi-carrier system with $n$ independent subchannels, we will no longer distinguish between subcarriers and chunks in the following description. Conventional SCM scheme in [3], in which some extra bits are designated to convey the subcarrier permutation information, is chosen for comparison. In the simulations, those bits are mapped to several symbols drawn from the same constellation employed for data transmission and the resulting signaling cost is accounted for in terms of transmit power loss. In addition, the non-SCM scheme and the ideal SCM scheme that assumes perfect knowledge of subcarrier permutation without any transmit power loss are selected as benchmarks. To shed more light on the fundamental characteristics of all involved SCM schemes, we assume perfect time-frequency synchronization, perfect CSI, and the optimal ML detection unless otherwise specified.

\section{A. Non-adaptive Modulation}

In this subsection, we focus on the performance comparison assuming non-adaptive modulation, namely all subcarriers employ the same modulation order. Both SCM types of BTB and BTW are taken into account in the comparison.

Fig. 5 presents the comparison results for $n=2$ and BPSK, where all schemes achieve an SE of $1 \mathrm{bps} / \mathrm{Hz}$. Comparing

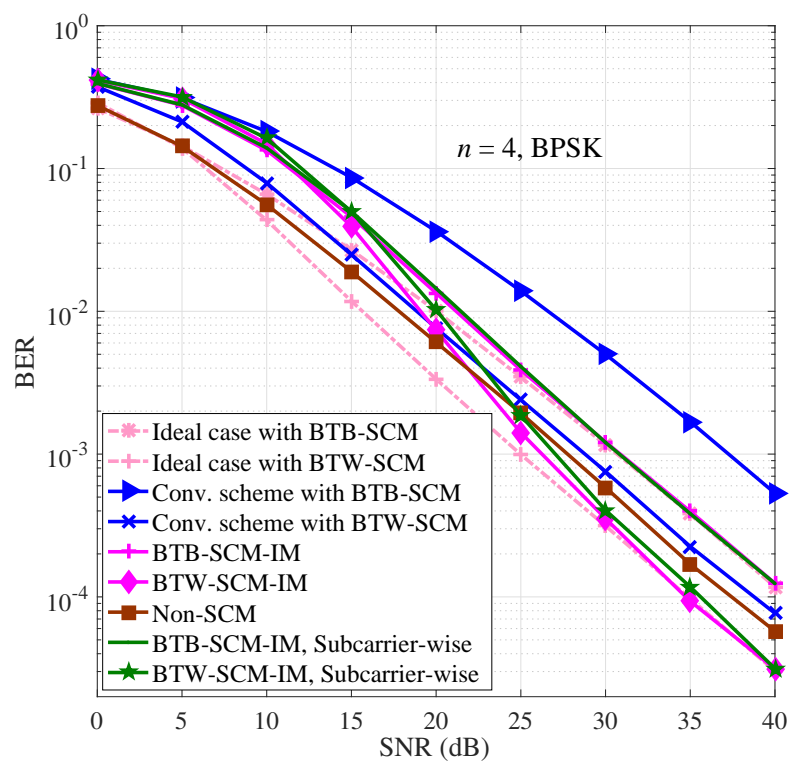

Fig. 6. Performance comparison among different (non-) SCM schemes with $n=4$ and BPSK considering both BTB and BTW.

both ideal SCM schemes with the non-SCM scheme, we observe that ideal BTW SCM always performs better than ideal BTB SCM in a large range of SNRs, corroborating the conclusions in [16]. However, the practical implementation of SCM with the conventional SCM schemes results in an apparent performance loss though the superiority of BTW SCM still persists for SNR beyond $13 \mathrm{~dB}$. This performance loss can be accounted by two reasons: one is the error detection of the subcarrier permutation and the other is the cost of transmit power. On the other hand, it is clear from Fig. 5 that the SCM-IM schemes perform close to the ideal SCM schemes and outperform the conventional SCM counterparts at medium-to-high SNR. Specifically, SNR gains of up to $4 \mathrm{~dB}$ and $1 \mathrm{~dB}$ for BTB and BTW, respectively, are obtained in the asymptotic SNR region. This advantage can be attributed to the nearly transparent delivery of the subcarrier permutation via the IM bits with second-order-diversity error protection [18]. Finally, as can be observed from Fig. 5, the derived upper bound is asymptotically tight.

Fig. 6 gives the comparison results when $n$ is increased from 2 to 4 . The performance of the subcarrier-wise detector for the SCM-IM schemes is presented, and the upper bounds have been removed for the clarity of the figure. Note that since we keep employing BPSK, the SE of $1 \mathrm{bps} / \mathrm{Hz}$ is retained for all schemes. Comparing Fig. 6 with Fig. 5, we observe that the performance of the non-SCM scheme remains unchanged while that of the ideal BTW SCM scheme is slightly improved especially at high SNR. This phenomenon can be explained by the fact that the non-SCM scheme employs parallel independent signal detection whose performance depends on the modulation type only, while the ideal BTW SCM scheme can better exploit the variation among dual-hop channels with increasing number of subcarriers. Additionally, from the comparison between two figures, we see that the 


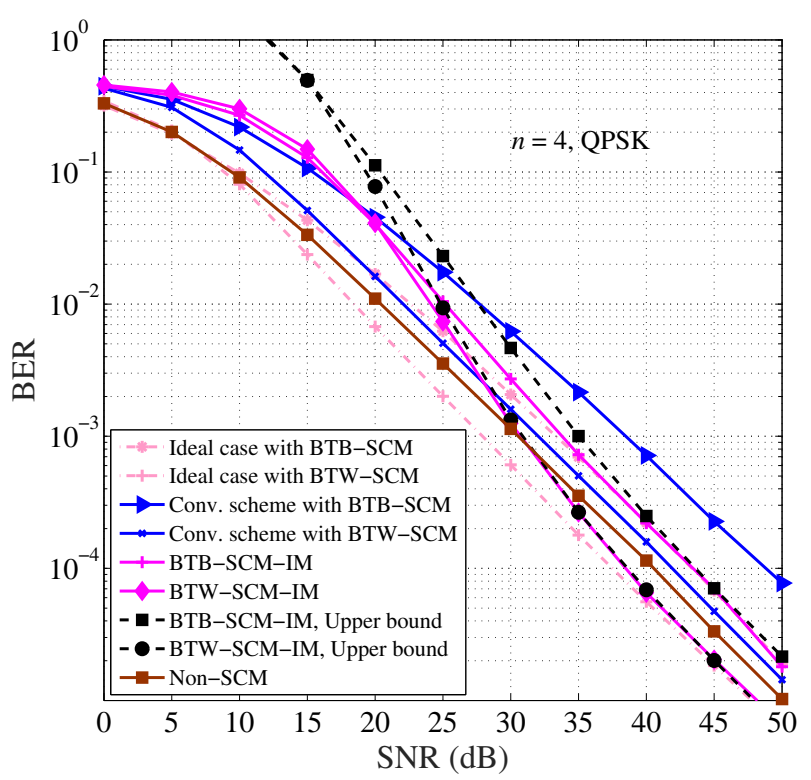

Fig. 7. Performance comparison among different (non-) SCM schemes with $n=4$ and QPSK considering both BTB and BTW.

performance of the conventional SCM scheme with either BTB or BTW is seriously deteriorated, and the conventional BTW-SCM scheme performs even worse than the non-SCM scheme. This is understandable since in this case the subcarrier permutation is encoded with a number of bits (equal to $\left\lceil\log _{2}(4 !)\right\rceil=5$ ) larger than that of the data symbols (equal to 4 ), occupying more than half transmit power. On the contrary, for the same reason clarified earlier, the performances of the SCM-IM schemes still asymptotically approach those of the ideal SCM schemes, and the SCM-IM scheme with BTW preserves its superiority over the non-SCM scheme for SNR beyond $22 \mathrm{~dB}$. Moreover, the subcarrier-wise detector has the capability of achieving near-optimal performance at low and high SNR values. The performance loss is confined within 1 $\mathrm{dB}$ in comparison with the ML detector in the medium SNR region.

Figs. 7 and 8 show the comparison results when the modulation order grows from 2 to 4 and further to 8 , such that the SE of all schemes is increased to $2 \mathrm{bps} / \mathrm{Hz}$ and further to $3 \mathrm{bps} / \mathrm{Hz}$, respectively. As anticipated, all schemes perform worse with the increase of the modulation order. From the comparison with Figs. 7 and 8 , we notice a crossing point of the BER curves of the SCM-IM scheme and the conventional scheme for either BTB or BTW, which shifts to a larger SNR as the modulation order increases. This can be explained by the fact that the phase rotation angle diminishes with the increase of the modulation order, leading to a smaller intra-mode distance that is defined as the minimum Euclidean distance between any two constellation points belonging to two different modes, and subsequently to a worse performance at low-to-medium SNR [18]. Fortunately, the crossing points correspond to BER values less than or equal to $10^{-3}$ that can be easily guaranteed in current wireless communications systems. Note that as before there is also a crossing point of the BER curves of

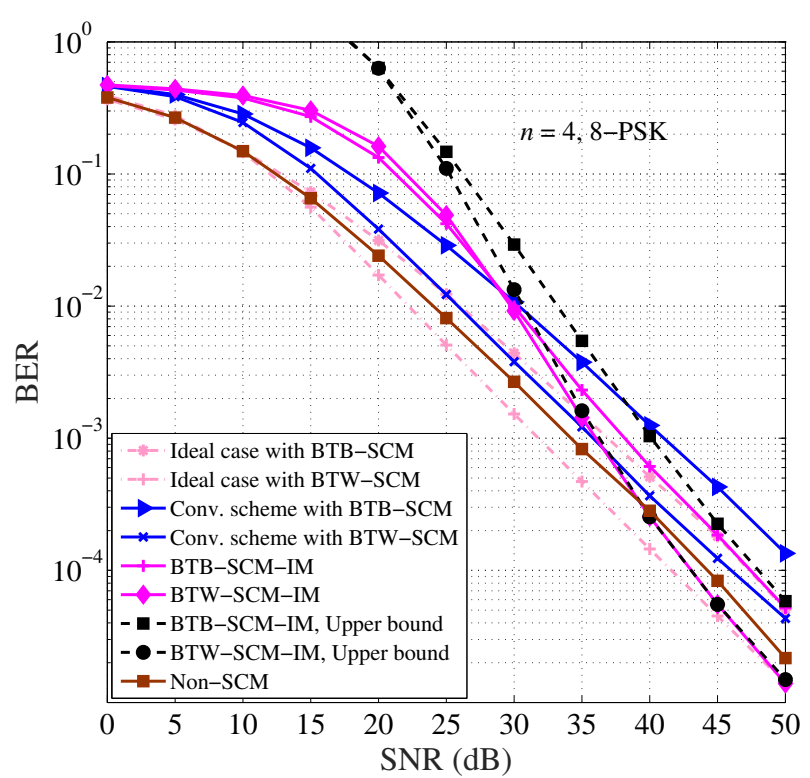

Fig. 8. Performance comparison among different (non-) SCM schemes with $n=4$ and 8 -PSK considering both BTB and BTW.

the SCM-IM scheme with BTW and the non-SCM scheme, but it lies in the BER zone on the order of $10^{-3}$ despite its shifting to a higher SNR value. Finally, consistent with the observations in Figs. 5 and 6, the performances of the SCMIM schemes coincide with those of the ideal schemes and the derived upper bounds are tight at high SNR.

By carefully observing Figs. 5-8, one can always discover an intersection point for the BER curves of the two SCMIM schemes. It is shown that the performance of the SCMIM scheme with BTB overwhelms that with BTW at the beginning, but becomes worse gradually. This can be proved rigorously based on the majorization theory by following the steps in [16]. Also, we can explain this phenomenon intuitively from the fact that the end-to-end channel gains of the paired subcarriers are spread after BTB SCM, but equalized after BTW SCM compared to the non-SCM case as follows. As SNR goes to infinity, the Gaussian $Q$-function with respect to the channel gain behaves more and more like a delta function at the origin with decreasing amplitude [36], such that the errors resulting from several worst subcarrier pairs in the BTB case will gradually dominate its BER, and the BER of the BTW case as well. On the contrary, as SNR goes to zero, the function becomes more and more flat near the origin, such that the error resulting from each subcarrier pair in the BTW case will become equal to that in the BTB case except several best subcarrier pairs, which lead to negligible BER since their channel gains may escape from the flat zone. To clearly show the superiority of the SCM-IM schemes, we summarize the SNR gains achieved by the SCM-IM schemes over the conventional SCM schemes in Table I for BTB SCM and BTW SCM with different values of $n$ and $M$. 
TABLE I

SNR GAINS ACHIEVED BY THE SCM-IM SCHEMES OVER THE CONVENTIONAL SCM SCHEMES FOR BTB SCM AND BTW SCM WITH DIFFERENT VALUES OF $n$ AND $M$.

\begin{tabular}{|c|c|c|}
\hline \multirow{2}{*}{$(n, M)$} & \multicolumn{2}{|c|}{ SNR Gain } \\
\cline { 2 - 3 } & BTB $\left(\mathrm{BER}=10^{-3}\right)$ & BTW $\left(\mathrm{BER}=10^{-4}\right)$ \\
\hline \hline$(2,2)$ & $3 \mathrm{~dB}$ & $1 \mathrm{~dB}$ \\
\hline$(4,2)$ & $6.5 \mathrm{~dB}$ & $4 \mathrm{~dB}$ \\
\hline$(4,4)$ & $5 \mathrm{~dB}$ & $3 \mathrm{~dB}$ \\
\hline$(4,8)$ & $3 \mathrm{~dB}$ & $3 \mathrm{~dB}$ \\
\hline
\end{tabular}

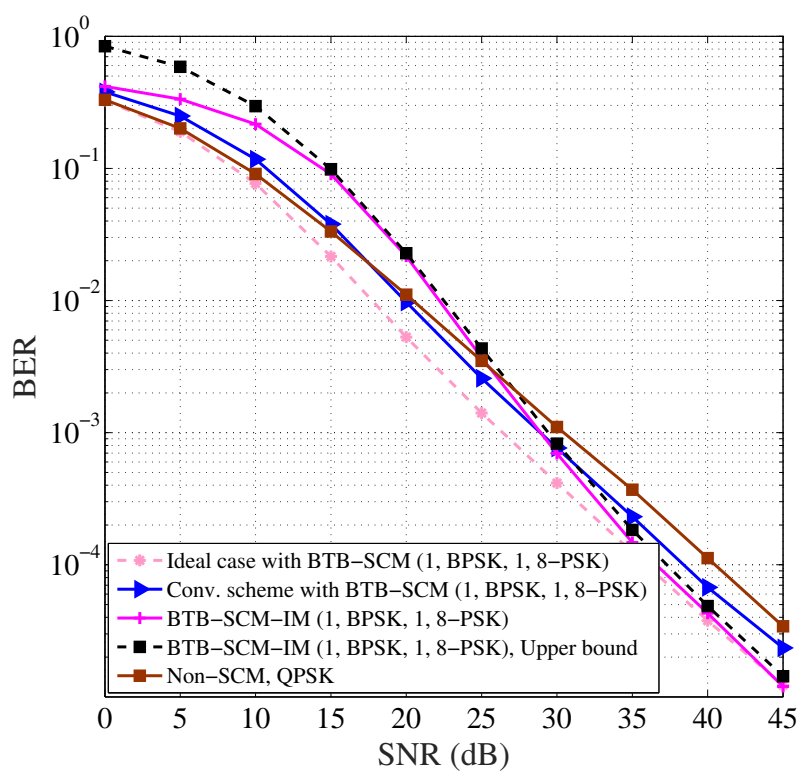

Fig. 9. Performance comparison among different (non-) SCM schemes with (1, BPSK, 1, 8-PSK) considering BTB.

\section{B. Adaptive Modulation}

In this subsection, we focus on the performance comparison assuming adaptive modulation, where a higher modulation order is employed for a better subcarrier and vice versa. In the simulations, we adopt the SCM type of BTB only since it is the proven best SCM solution in achieving the maximum information rate. Furthermore, we consider the following two different parameter configurations corresponding to $n=2$ and $n=4$, respectively:

i) (1, BPSK, 1, 8-PSK), referring to the case that the best subcarrier pair transmits an 8-PSK symbol while the worst subcarrier pair transmits a BPSK symbol;

ii) (2, BPSK, 2, 8-PSK), referring to the case that the first two best subcarrier pairs employ 8-PSK while the first two worst subcarrier pairs employ BPSK.

For both configurations, all SCM schemes achieve an SE of $2 \mathrm{bps} / \mathrm{Hz}$. Therefore, to ensure fair comparison, we assume QPSK for the non-SCM scheme.

Fig. 9 illustrates the comparison results for configuration i). It is obvious from Fig. 9 that unlike non-adaptive modulation cases, in the case of adaptive modulation the performance of the ideal BTB SCM scheme overwhelms that of the non-SCM scheme. This can be understood from the capability of BTB SCM in achieving the channel capacity. On the other hand,

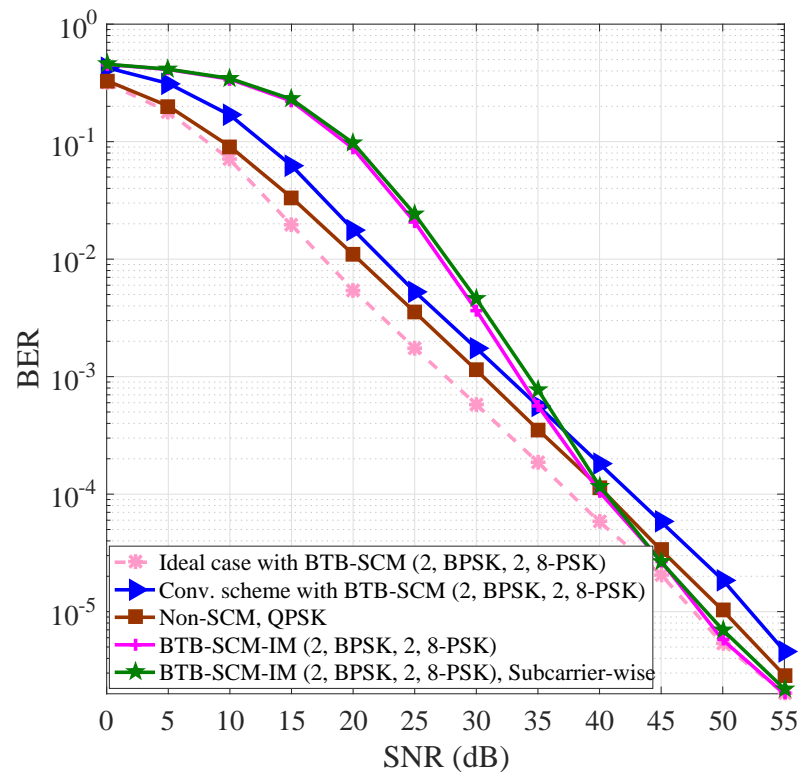

Fig. 10. Performance comparison among different (non-) SCM schemes with (2, BPSK, 2, 8-PSK) considering BTB.

despite a performance loss with respect to the ideal case due to the potential error in the detection of the SCM information, both practical SCM schemes can still outperform the non-SCM scheme in the medium-to-high SNR regime. The SCM-IM scheme performs worse than the conventional scheme at low SNR, but it rapidly catches up and finally approaches the ideal SCM scheme with the increase of SNR as always. Clearly, the derived upper bound is asymptotically tight, verifying its extensibility.

Fig. 10 depicts the comparison results for configuration ii). Here, we have included the BER curve of the subcarrierwise detector for the SCM-IM scheme, and deleted the derived upper bound for figure clarity. Similar to non-adaptive modulation cases, as $n$ increases to 4 , the performance of the conventional BTB SCM scheme is seriously degraded, becoming worse than that of the non-SCM scheme. However, the SCM-IM scheme with BTB is still superior to the nonSCM scheme at high SNR, performing nearly the same as the ideal BTB SCM scheme. Comparing Fig. 10 with Fig. 8, one can observe very similar performance trend. This is because in our design, the BPSK constellation is visualized as an 8PSK constellation with two practical constellation points for determining the phase rotation angle, which happens to be the same as that in Fig. 8. It is clear from Fig. 10 that the subcarrier-wise detector achieves near-optimal performance for the SCM-IM scheme, almost maintaining the advantage of SCM-IM in terms of BER.

Overall, we observe from Figs. 5-10 that the SCM-IM schemes perform close to the ideal SCM schemes and outperform the conventional SCM counterparts at medium-to-high SNR for both non-adaptive and adaptive modulation. Moreover, the BTW-SCM-IM scheme with non-adaptive modulation also performs better than the non-SCM scheme at medium-to-high SNR. 


\section{CONCLUSION}

In this paper, we have addressed the problem of reordering information conveying in relaying systems with SCM, and proposed a practical solution, namely SCM-IM, that applies to both adaptive and non-adaptive modulation scenarios. The proposed SCM-IM scheme employs phase rotation on the received signal at the relay to create multiple distinguishable modes adaptively, and transfers the reordering information to the mode permutation information that is embedded into the forwarded signal at the relay, completely eliminating the signaling overhead. By isolating the errors arising from mode permutation detection and conventional modulation symbol detection, we derived an asymptotically tight BER upper bound in closed form and further characterized the diversity gain for the proposed SCM-IM schemes with both BTB and BTW. Monte Carlo simulations have been performed to investigate the BER performance of the proposed SCM schemes. It is shown that the proposed SCM schemes can achieve up to $6.5 \mathrm{~dB}$ and $4 \mathrm{~dB}$ SNR gains for BTB and BTW cases, respectively, compared with the conventional SCM scheme, and even perform close to the ideal SCM scheme with the perfect knowledge of the reordering information at high SNR. The intuition behind our results is that the stronger error protection for the mode permutation, which is proved to yield second-order diversity, makes the proposed SCM-IM schemes superior to conventional SCM schemes and close to the ideal SCM scheme. Finally, it is worth noting that though the SCM-IM scheme is introduced to an OFDM dual-hop AF relaying system merely in this paper, its principle is general and applicable to multi-antenna and multi-hop settings as well as their combination.

\section{APPENDIX A}

\section{PROOF OF LEMMA 1}

According to the union bounding technique, $P_{b 1}$ is upper bounded by

$$
\begin{aligned}
P_{b 1} \leq & \frac{1}{p 2^{p} n !} \sum_{i=1}^{n !} \sum_{j=1, j \neq i}^{n !} \operatorname{Pr}\left(I_{i} \rightarrow I_{j}\right) \\
& \times \sum_{\mathbf{x}} \sum_{\hat{\mathbf{x}}} \operatorname{Pr}\left(\mathbf{x} \rightarrow \hat{\mathbf{x}} \mid I_{i} \rightarrow I_{j}\right) N(\mathbf{x}, \hat{\mathbf{x}}),
\end{aligned}
$$

where $\operatorname{Pr}\left(\mathbf{x} \rightarrow \hat{\mathbf{x}} \mid I_{i} \rightarrow I_{j}\right)$ represents the pairwise error probability (PEP) of the event that the transmitted $\mathrm{x}$ is erroneously detected as $\hat{\mathbf{x}}$ conditioned on the mode permutation detection error $I_{i} \rightarrow I_{j}$, and $N(\mathbf{x}, \hat{\mathbf{x}})$ denotes the number of erroneous bits when $\mathbf{x}$ is detected as $\hat{\mathbf{x}}$.

With $D\left(I_{i}, I_{j}\right)+\bar{D}\left(I_{i}, I_{j}\right)=n$, (22) can be rewritten as (23), shown at the top of the next page, where

$$
T_{1}=\frac{1}{2^{p}} \sum_{\mathbf{x}} \sum_{\hat{\mathbf{x}}} \operatorname{Pr}\left(X_{m} \rightarrow \hat{X}_{m} \mid I_{i} \rightarrow I_{j}\right) N\left(X_{m}, \hat{X}_{m}\right)
$$

and

$$
T_{2}=\frac{1}{2^{p}} \sum_{\mathbf{x}} \sum_{\hat{\mathbf{x}}} \operatorname{Pr}\left(X_{\kappa} \rightarrow \hat{X}_{\kappa} \mid I_{i} \rightarrow I_{j}\right) N\left(X_{\kappa}, \hat{X}_{\kappa}\right),
$$

are associated with the subcarriers whose modes are detected correctly and incorrectly, respectively. It can be figured out that $T_{1}$ is the symbol error probability of OFDM AF relaying with $M$-PSK and ideal SCM, which is given by

$$
T_{1} \approx P_{b-S C M}(M) \log _{2}(M) .
$$

As for $T_{2}$, with the erroneously detected mode, the probability of estimating a bit correctly is about $1 / 2$, and the probability that $\log _{2}(M)$ information bits are correctly detected is about $(1 / 2)^{\log _{2}(M)}$. Hence, we arrive at

$$
T_{2} \approx\left(1-(1 / 2)^{\log _{2}(M)}\right) \log _{2}(M) .
$$

Consequently, putting (26) and (27) into (23) yields (8). Moreover, since all signal points from $n$ modes constitute the regular $n M$-PSK constellation and the error probability of detecting one mode can be regarded as the symbol error probability of the $n M$-PSK constellation, $\operatorname{Pr}\left(I_{i} \rightarrow I_{j}\right)$ can be expressed as (9), thus completing the proof. Note that the above derivation of $P_{b 1}$ is applicable to both BTB and BTW SCM schemes.

\section{APPENDIX B \\ PROOF OF LEMMA 2}

Given the PDF of the end-to-end SNR on the $k$-th subcarrier pair, i.e., $f_{\gamma_{k}}\left(\gamma_{k}\right)$, the BEP on the $k$-th subcarrier pair can be given by

$$
P_{b-S C M}(M, k)=\int_{0}^{\infty} P_{b-S C M \mid \gamma_{k}}(M, k) f_{\gamma_{k}}\left(\gamma_{k}\right) d \gamma_{k},
$$

where $P_{b-S C M \mid \gamma_{k}}(M, k)$ is the BEP of $M$-PSK on the $k$ th subcarrier pair conditioned on $\gamma_{k}$. For any value of $M$, $P_{b-S C M \mid \gamma_{k}}(M, k)$ can be given in the form of [33, Eq. (8.33)]

$$
P_{b-S C M \mid \gamma_{k}}(M, k) \cong A Q\left(\sqrt{B \gamma_{k}}\right),
$$

where $B=2 \sin ^{2}(\pi / M)$, and $A=1$ for $M=2$, while $A=2 / \log _{2}(M)$ for $M \geq 4$. By utilizing the well-known approximation of $Q$-function, namely

$$
Q(x) \approx \frac{1}{12} e^{-\frac{1}{2} x^{2}}+\frac{1}{4} e^{-\frac{2}{3} x^{2}},
$$

(28) can be simplified as

$$
\begin{aligned}
& P_{b-S C M}(M, k) \\
& \approx \int_{0}^{\infty}\left(\frac{A}{12} \exp \left(-\frac{B \gamma_{k}}{2}\right)+\frac{A}{4} \exp \left(-\frac{2 B \gamma_{k}}{3}\right)\right) f_{\gamma_{k}}\left(\gamma_{k}\right) d \gamma_{k} \\
& =\frac{A}{12} \mathcal{M}_{\gamma_{k}}\left(\frac{B}{2}\right)+\frac{A}{4} \mathcal{M}_{\gamma_{k}}\left(\frac{2 B}{3}\right)
\end{aligned}
$$

where $\mathcal{M}_{\gamma_{k}}(\cdot)$ is the moment generating function (MGF) of $\gamma_{k}$. Next, we will deal with the calculation of $\mathcal{M}_{\gamma_{k}}(\cdot)$ in the BTB and BTW SCM scenarios, respectively.

\section{A. BTB SCM}

Assuming that the subcarriers of both base station $\rightarrow$ relay and realy $\rightarrow$ mobile station links are increasingly ordered according to the magnitude of their transfer functions, the instantaneous end-to-end SNR on the $k$-th subcarrier pair can be given by

$$
\gamma_{k}=\frac{\gamma_{1, k} \gamma_{2, k}}{\gamma_{2, k}+C}=\frac{\gamma_{1, k}}{1+C d_{k}}
$$




$$
\begin{aligned}
& P_{b 1} \approx \frac{1}{p 2^{p} n !} \sum_{i=1}^{n !} \sum_{j=1, j \neq i}^{n !} \operatorname{Pr}\left(I_{i} \rightarrow I_{j}\right) \sum_{\mathbf{x}} \sum_{\hat{\mathbf{x}}} \sum_{k=1}^{n} \operatorname{Pr}\left(X_{k} \rightarrow \hat{X}_{k} \mid I_{i} \rightarrow I_{j}\right) N\left(X_{k}, \hat{X}_{k}\right) \\
&=\frac{1}{p 2^{p} n !} \sum_{i=1}^{n !} \sum_{\substack{j=1 \\
j \neq i}}^{n !} \operatorname{Pr}\left(I_{i} \rightarrow I_{j}\right) \sum_{\mathbf{x}} \sum_{\hat{\mathbf{x}}}\left[\sum_{m=1}^{D\left(I_{i}, I_{j}\right)} \operatorname{Pr}\left(X_{m} \rightarrow \hat{X}_{m} \mid I_{i} \rightarrow I_{j}\right) N\left(X_{m}, \hat{X}_{m}\right)\right. \\
&\left.\quad+\sum_{\kappa=1}^{\bar{D}\left(I_{i}, I_{j}\right)} \operatorname{Pr}\left(X_{\kappa} \rightarrow \hat{X}_{\kappa} \mid I_{i} \rightarrow I_{j}\right) N\left(X_{\kappa}, \hat{X}_{\kappa}\right)\right] \\
&=\frac{1}{p n !} \sum_{i=1}^{n !} \sum_{j=1, j \neq i}^{n !} \operatorname{Pr}\left(I_{i} \rightarrow I_{j}\right)\left[\sum_{m=1}^{D\left(I_{i}, I_{j}\right)} T_{1}+\sum_{\kappa=1}^{\bar{D}\left(I_{i}, I_{j}\right)} T_{2}\right]
\end{aligned}
$$

where $d_{k}=1 / \gamma_{2, k}$. The PDF of $d_{k}$ is [17]

$$
f_{d_{k}}\left(d_{k}\right)=\frac{1}{d_{k}^{2}} f_{2, k}^{w}\left(\frac{1}{d_{k}}\right)
$$

where

$$
f_{2, k}^{w}(x)=\sum_{i=0}^{k-1} \frac{a_{i}}{\bar{\gamma}_{2}} \exp \left(-\frac{b_{i}}{\bar{\gamma}_{2}} x\right)
$$

is the PDF of the SNR on the $k$-th weakest subcarrier out of $n$ subcarriers in the second hop with

$$
\begin{aligned}
a_{i} & =(-1)^{i} n C(n-1, k-1) C(k-1, i), \\
b_{i} & =i+n-k+1,
\end{aligned}
$$

and

$$
\bar{\gamma}_{2}=\varepsilon_{R} \Omega_{2} / N_{02} .
$$

The PDF of the denominator in (32), denoted by $z_{k}=1+$ $C d_{k}$, can be derived as

$$
f_{z_{k}}\left(z_{k}\right)=C \frac{\mathcal{U}\left(z_{k}-1\right)}{\left(z_{k}-1\right)^{2}} \sum_{i=0}^{k-1} \frac{a_{i}}{\bar{\gamma}_{2}} \exp \left(-\frac{C b_{i}}{\bar{\gamma}_{2}\left(z_{k}-1\right)}\right) \text {. }
$$

From (32) and (37), the PDF of $\gamma_{k}$ can be calculated as

$$
f_{\gamma_{k}}^{B T B}(x)=\int_{0}^{\infty} z_{k} f_{1, k}^{w}\left(x z_{k}\right) f_{z_{k}}\left(z_{k}\right) d z_{k},
$$

where $f_{1, k}^{w}(\cdot)$ denotes the PDF of the SNR on the $k$-th weakest subcarrier in the first hop. Similar to $f_{2, k}^{w}(\cdot), f_{1, k}^{w}(\cdot)$ can be expressed as [34]

$$
f_{1, k}^{w}(x)=\sum_{i=0}^{k-1} \frac{a_{i}}{\bar{\gamma}_{1}} \exp \left(-\frac{b_{i}}{\bar{\gamma}_{1}} x\right) .
$$

By substituting (37) and (39) into (38), we have

$$
\begin{aligned}
f_{\gamma_{k}}^{B T B}(x)= & \frac{C}{\bar{\gamma}_{1} \bar{\gamma}_{2}} \sum_{j=0}^{k-1} \sum_{i=0}^{k-1} a_{j} a_{i} \\
& \times \int_{1}^{\infty} \frac{z_{k}}{\left(z_{k}-1\right)^{2}} \exp \left(-\frac{b_{j} z_{k} x}{\bar{\gamma}_{1}}-\frac{b_{i} C}{\bar{\gamma}_{2}\left(z_{k}-1\right)}\right) d z_{k} .
\end{aligned}
$$

Resorting to [35, Eq. (3.471-12)], (40) reduces to

$$
\begin{aligned}
f_{\gamma_{k}}^{B T B}(x)= & \frac{2}{\bar{\gamma}_{1}} \sum_{j=0}^{k-1} \sum_{i=0}^{k-1} a_{j} a_{i} \exp \left(-\frac{b_{j}}{\bar{\gamma}_{1}} x\right)\left[\sqrt{\frac{C b_{j} x}{b_{i} \bar{\gamma}_{1} \bar{\gamma}_{2}}}\right. \\
& \left.\times K_{1}\left(2 \sqrt{\frac{C b_{j} b_{i} x}{\bar{\gamma}_{1} \bar{\gamma}_{2}}}\right)+\frac{C}{\bar{\gamma}_{2}} K_{0}\left(2 \sqrt{\frac{C b_{j} b_{i} x}{\bar{\gamma}_{1} \bar{\gamma}_{2}}}\right)\right] .
\end{aligned}
$$

From (41), the MGF of $\gamma_{k}$ can be presented as [35, Eqs. (13.6.28), (13.6.30), (6.5.19)]

$$
\begin{aligned}
\mathcal{M}_{\gamma_{k}}^{B T B}(s)= & \int_{0}^{\infty} e^{-s \gamma_{k}} f_{\gamma_{k}}^{B T B}\left(\gamma_{k}\right) d \gamma_{k} \\
= & \frac{1}{\bar{\gamma}_{1}} \sum_{j=0}^{k-1} \sum_{i=0}^{k-1} \frac{a_{j} a_{i}}{V_{j}(s)}\left[\frac{1}{b_{i}}-\exp \left(\frac{C A_{j, i}}{V_{j}(s)}\right)\right. \\
& \left.\times \operatorname{Ei}\left(-\frac{C A_{j, i}}{V_{j}(s)}\right)\left(\frac{C}{\bar{\gamma}_{2}}-\frac{C A_{j, i}}{b_{i} V_{j}(s)}\right)\right],
\end{aligned}
$$

where $V_{j}(s)=s+b_{j} / \bar{\gamma}_{1}$ and $A_{j, i}=b_{j} b_{i} / \bar{\gamma}_{1} \bar{\gamma}_{2}$.

\section{B. BTW SCM}

In this scenario, the $k$-th weakest subcarrier out of $n$ subcarriers in the first hop is mapped to the $k$-th strongest subcarrier in the second hop. Through a similar approach as that in the BTB SCM scenario, the PDF of SNR on the $k$-th subcarrier pair in the BTW SCM scenario becomes

$$
\begin{aligned}
f_{\gamma_{k}}^{B T W}(x)= & \frac{2}{\bar{\gamma}_{1}} \sum_{j=0}^{k-1} \sum_{i=0}^{N-k} a_{j} \delta_{i} \exp \left(-\frac{b_{j}}{\bar{\gamma}_{1}} x\right)\left[\sqrt{\frac{C b_{j} x}{\varsigma_{i} \bar{\gamma}_{1} \bar{\gamma}_{2}}}\right. \\
& \left.\times K_{1}\left(2 \sqrt{\frac{C b_{j} \varsigma_{i} x}{\bar{\gamma}_{1} \bar{\gamma}_{2}}}\right)+\frac{C}{\bar{\gamma}_{2}} K_{0}\left(2 \sqrt{\frac{C b_{j} \varsigma_{i} x}{\bar{\gamma}_{1} \bar{\gamma}_{2}}}\right)\right],
\end{aligned}
$$

where $\delta_{i}=(-1)^{i} n C(n-1, k-1) C(n-k, i)$ and $\varsigma_{i}=i+k$. Similar to (42), the MGF of $\gamma_{k}$ for the BTW SCM can be given by

$$
\begin{aligned}
\mathcal{M}_{\gamma_{k}}^{B T W}(s)= & \frac{1}{\bar{\gamma}_{1}} \sum_{j=0}^{k-1} \sum_{i=0}^{n-k} \frac{a_{j} \delta_{i}}{V_{j}(s)}\left[\frac{1}{\varsigma_{i}}-\exp \left(\frac{C B_{j, i}}{V_{j}(s)}\right)\right. \\
& \left.\times \operatorname{Ei}\left(-\frac{C B_{j, i}}{V_{j}(s)}\right)\left(\frac{C}{\bar{\gamma}_{2}}-\frac{C B_{j, i}}{\varsigma_{i} V_{j}(s)}\right)\right],
\end{aligned}
$$


where $B_{j, i}=b_{j} \varsigma_{i} / \bar{\gamma}_{1} \bar{\gamma}_{2}$.

Finally, by substituting (42) or (44) into (31), and averaging $P_{b-S C M}(M, k)$ over $n$ subcarriers, we complete the proof.

\section{APPENDIX C \\ PROOF OF PROPOSITION}

According to the union bounding technique, an upper bound on the overall BER of OFDM AF relaying with SCM-IM can be expressed as

$$
P_{b} \leq \frac{1}{p 2^{p}} \sum_{\mathbf{x}} \sum_{\hat{\mathbf{x}}} \operatorname{Pr}(\mathbf{x} \rightarrow \hat{\mathbf{x}}) N(\mathbf{x}, \hat{\mathbf{x}}) .
$$

Considering that there are $n$ ! possible mode permutations, $\operatorname{Pr}(\mathbf{x} \rightarrow \hat{\mathbf{x}})$ can be upper bounded by

$$
\begin{aligned}
\operatorname{Pr}(\mathbf{x} \rightarrow \hat{\mathbf{x}}) & \leq \frac{1}{n !} \sum_{i=1}^{n !} \sum_{j=1}^{n !} \operatorname{Pr}\left(\mathbf{x} \rightarrow \hat{\mathbf{x}}, I_{i} \rightarrow I_{j}\right) \\
& =\frac{1}{n !} \sum_{i=1}^{n !} \sum_{j=1}^{n !} \operatorname{Pr}\left(\mathbf{x} \rightarrow \hat{\mathbf{x}} \mid I_{i} \rightarrow I_{j}\right) \operatorname{Pr}\left(I_{i} \rightarrow I_{j}\right) .
\end{aligned}
$$

Therefore, substituting (46) into (45) yields

$$
\begin{aligned}
P_{b} \leq & \frac{1}{p 2^{p} n !} \sum_{\mathbf{x}} \sum_{\hat{\mathbf{x}}} \sum_{i=1}^{n !} \sum_{j=1}^{n !} \operatorname{Pr}\left(\mathbf{x} \rightarrow \hat{\mathbf{x}} \mid I_{i} \rightarrow I_{j}\right) \operatorname{Pr}\left(I_{i} \rightarrow I_{j}\right) N(\mathbf{x}, \hat{\mathbf{x}}) \\
= & \frac{1}{p 2^{p} n !} \sum_{\mathbf{x}} \sum_{\hat{\mathbf{x}}}\left[\sum_{i=1}^{n !} \sum_{j=1, j \neq i}^{n !} \operatorname{Pr}\left(\mathbf{x} \rightarrow \hat{\mathbf{x}} \mid I_{i} \rightarrow I_{j}\right) N(\mathbf{x}, \hat{\mathbf{x}})\right. \\
& \left.\times \operatorname{Pr}\left(I_{i} \rightarrow I_{j}\right)+\sum_{i=1}^{n !} \operatorname{Pr}\left(\mathbf{x} \rightarrow \hat{\mathbf{x}} \mid I_{i} \rightarrow I_{i}\right) \operatorname{Pr}\left(I_{i} \rightarrow I_{i}\right) N(\mathbf{x}, \hat{\mathbf{x}})\right] \\
\leq & P_{b 1}+\frac{1}{p 2^{p}} \sum_{\mathbf{x}} \sum_{\hat{\mathbf{x}}} \operatorname{Pr}\left(\mathbf{x} \rightarrow \hat{\mathbf{x}} \mid I_{i} \rightarrow I_{i}\right) N(\mathbf{x}, \hat{\mathbf{x}}), \quad(47)
\end{aligned}
$$

where the fact that $0 \leq \operatorname{Pr}\left(I_{i} \rightarrow I_{i}\right) \leq 1$ is considered. We observe that the second term on the right side of (47) is actually the BEP of OFDM AF relay systems with perfect (error-free) SCM detection, i.e., $P_{b-S C M}(M)$, thus completing the proof.

\section{APPENDIX D}

\section{PROOF OF LEMMA 3}

Since in the adaptive scenario the minimum Euclidean distance between any two modes is greater than or equal to that of the $n M_{m}$-ary PSK constellation, the probability of erroneously detecting a mode is smaller than or equal to the symbol error rate of $n M_{m}$-ary PSK, verifying (19). Noting $p=\sum_{k=1}^{n} \log _{2}\left(M_{k}\right)$, from (22)-(27) we have (19) and (20), completing the proof.

\section{REFERENCES}

[1] A. Hottinen and T. Heikkinen, "Subchannel assignment in OFDM relay nodes," in Proc. 40th Annual Conf. Inf. Sci. and Syst. (CISS), Princeton, NJ, USA, Mar. 2006, pp. 1314-1317.

[2] I. Hammerstrom and A. Wittneben, "Joint power allocation for nonregenerative MIMO-OFDM relay links," in Proc. IEEE Int. Conf. Acoust, Speech, and Sign. Process. (ICASSP), Toulouse, France, May 2006, pp. IV-49-IV-52.

[3] M. Herdin, "A chunk based OFDM amplify-and-forward relaying scheme for 4G mobile radio systems," in Proc. IEEE Int. Conf. Commun. (ICC), Istanbul, Turkey, Jun. 2006, pp. 4507-4512.
[4] I. Hammerstrom and A. Wittneben, "On the optimal power allocation for nonregenerative OFDM relay links," in Proc. IEEE Int. Conf. Commun. (ICC), Istanbul, Turkey, Jun. 2006, pp. 4463-4468.

[5] A. Hottinen and T. Heikkinen, "Optimal subchannel assignment in a two-hop OFDM relay," in Proc. IEEE 8th Workshop Sign. Proc. Adv. in Wireless Commun. (SPAWC), Istanbul, Turkey, Jun. 2007, pp. 1-5.

[6] C. R. N. Athaudage, M. Saito, and J. Evans, "Performance analysis of dual-hop OFDM relay systems with subcarrier mapping," in Proc. IEEE Int. Conf. Commun. (ICC), Beijing, China, May 2008, pp. 4419-4423.

[7] N. Zhou, X. Zhu, Y. Huang, and H. Lin, "Adaptive resource allocation for multi-destination relay systems based on OFDM modulation," in Proc. IEEE Int. Conf. Commun. (ICC), pp. 1-5, Dresden, Germany, Jun. 2009.

[8] Y. Li, W. Wang, J. Kong, and M. Peng, "Subcarrier pairing for amplify-and-forward and decode-and-forward OFDM relay links," IEEE Commun. Lett., vol. 13, no. 4, pp. 209-211, Apr. 2009

[9] W. Wang, S. Yan, and S. Yang, "Optimally joint subcarrier matching and power allocation in OFDM multihop system," EURASIP J. Adv. Signal Process., vol. 2008, pp. 1-8, 2008.

[10] W. Wang and R. Wu, "Capacity maximization for OFDM two-hop relay system with separate power constraints," IEEE Trans. Veh. Tech., vol. 58 , no. 9, pp. 4943-4954, Dec. 2009.

[11] Y. Li, W. Wang, J. Kong, W. Hong, X. Zhang, and M. Peng, "Power allocation and subcarrier pairing in OFDM-based relaying networks," in Proc. IEEE Int. Conf. Commun. (ICC), Beijing, China, May 2008, pp. 2602-2606.

[12] C.-N. Hsu, H.-J. Su, and P.-H. Lin, "Joint subcarrier pairing and power allocation for OFDM transmission with decode-and-forward relaying," IEEE Trans. Signal Process., vol. 59, no. 1, pp. 399-414, Jan. 2011.

[13] M. Hajiaghayi, M. Dong, and B. Liang, "Jointly optimal channel pairing and power allocation for multichannel multihop relaying," IEEE Trans. Signal Process., vol. 59, no. 10, pp. 4998-5012, Oct. 2011.

[14] S. Chen, F. Liu, X. Zhang, C. Xiong, and D. Yang, "Symbol error performance analysis of OFDM relaying system with subcarrier mapping scheme," IEEE Commun. Lett., vol. 14, no. 7, pp. 638-640, Jul. 2010.

[15] R. A. Shah, N. Rajatheva, and Y. Ji, "Analysis of BER and Capacity for dual-hop OFDM relay system with subcarrier mapping in Nakagami- $m$ fading," in Proc. IEEE Int. Conf. Commun. (ICC), Sydney, Australia, Jun. 2014, pp. 5089-5094.

[16] C. K. Ho and A. Pandharipande, "BER minimization in relay-assisted OFDM systems by subcarrier permutation," in Proc. IEEE Veh. Tech. Conf. (VTC), Singapore, May 2008, pp. 1489-1493.

[17] E. Kocan, M. Pejanovic-Djurisic, D. S. Michalopoulos, and G. K. Karagiannidis, "Performance evaluation of OFDM amplify-and-forward relay system with subcarrier permutation," IEICE Trans. Commun., vol. E93-B, no. 5, pp. 1216-1223, May 2009.

[18] M. Wen, E. Basar, Q. Li, B. Zheng, and M. Zhang, "Multiple-mode orthogonal frequency division multiplexing with index modulation," IEEE Trans. Commun., vol. 65, no. 9, pp. 3892-3906, Sep. 2017.

[19] Q. Li, M. Wen, E. Basar, H. V. Poor, B. Zheng, and F. Chen, "Diversityenhancing multiple-mode OFDM with index modulation," IEEE Trans. Commun., vol. 66, no. 8, pp. 3653-3666, Aug. 2018.

[20] M. Wen, Q. Li, E. Basar, and W. Zhang, "Generalized multiple-mode OFDM with index modulation," IEEE Trans. Wireless Commun., vol. 17, no. 10, pp. 6531-6543, Oct. 2018.

[21] E. Basar, U. Aygolu, E. Panayirci, and H. V. Poor, "Orthogonal frequency division multiplexing with index modulation," IEEE Trans. Signal Process., vol. 61, no. 22, pp. 5536-5549, Nov. 2013.

[22] M. Wen, X. Cheng, M. Ma, B. Jiao, and H. V. Poor, "On the achievable rate of OFDM with index modulation," IEEE Trans. Signal Process. vol. 64, no. 8, pp. 1919-1932, Apr. 2016.

[23] M. Wen, B. Ye, E. Basar, Q. Li, and F. Ji, "Enhanced Orthogonal Frequency Division Multiplexing with Index Modulation," IEEE Trans. Wireless Commun., vol. 16, no. 7, pp. 4786-4801, Jul. 2017.

[24] T. Mao, Q. Wang, Z. Wang, and S. Chen, "Novel index modulation techniques: A survey," IEEE Commun. Survey \& Tut., vol. 21, no. 1, pp. 315-348, 1st Quarter 2019.

[25] E. Basar, "Index modulation techniques for 5G wireless networks," IEEE Commun. Mag., vol. 54, no. 7, pp. 168-175, Jul. 2016.

[26] X. Cheng, M. Zhang, M. Wen, and L. Yang, "Index modulation for 5G: Striving to do more with less," IEEE Wireless Commun., vol. 25, no. 2, pp. 126-132, Apr. 2018.

[27] E. Basar, M. Wen, R. Mesleh, M. Di Renzo, Y. Xiao, and H. Haas, "Index modulation techniques for next-generation wireless networks," IEEE Access, vol. 5, pp. 16693-16746, Sep. 2017. 
[28] M. O. Hasna and M.S. Alouini, "A performance study of dual-hop transmissions with fixed gain relays," IEEE Trans. Wireless Commun. vol. 3, no. 6, pp. 1963-1968, Nov. 2004.

[29] I. S. Gradshteyn and I. M. Ryzhik, Table of Integrals, Series, and Products, 7th ed., New York, NY, USA: Academic Press, 2007.

[30] J. Cavers, "Variable-rate transmission for Rayleigh fading channels," IEEE Trans. Commun., vol. 20, no. 1, pp. 15-22, Feb. 1972.

[31] A. J. Goldsmith, Wireless Communications. Cambridge University Press, 2005.

[32] Q. Li, M. Wen, H. V. Poor, and F. Chen, "Information guided precoding for OFDM," IEEE Access, vol. 5, pp. 19644-19656, Oct. 2017.

[33] M. K. Simon and M.-S. Alouini, Digital Communication Over Fading Channels, 2nd ed. New York, NY, USA: Wiley, 2005.

[34] A. Papoulis, Probability, Random Variables, and Stochastic Procceses. 3rd ed., McGraw-Hill, 1991.

[35] I. S. Gradshteyn and I. M. Ryzhik, Table of Integrals, Series, and Products. 6th ed., Academic, New York, 2000.

[36] Z. Wang and G. B. Giannakis, "A simple and general parameterization quantifying performance in fading channels," IEEE Trans. Commun., vol. 51, no. 8, pp. 1389-1398, Aug. 2003.

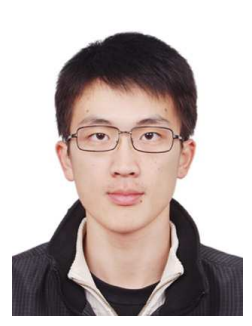

Miaowen Wen (M'14-SM'18) received the B.S. degree from Beijing Jiaotong University, Beijing, China, in 2009, and the Ph.D. degree from Peking University, Beijing, China, in 2014. From 2012 to 2013, he was a Visiting Student Research Collaborator with Princeton University, Princeton, NJ, USA. $\mathrm{He}$ is currently an Associate Professor with the South China University of Technology, Guangzhou, China. He has published a Springer book entitled Index Modulation for $5 G$ Wireless Communications and more than 110 research papers, which include more than 70 journal papers and more than 40 conference papers. His research interests include index modulation, non-orthogonal multiple access, physical layer security, and molecular communications.

Dr. Wen was the recipient of the Excellent Doctoral Dissertation Award from Peking University and the Best Paper Awards from the IEEE ITST'12, the IEEE ITSC'14, and the IEEE ICNC'16. He was recognized as an Exemplary Reviewer for the IEEE COMMUNICATIONS LETTERS in 2017. He served as a symposium co-chair for the IEEE ICNC'2019, a workshop co-chair for the IEEE/CIC ICCC'2018, and a Guest Editor for IEEE JSAC (Special Issue on Spatial Modulation for Emerging Wireless Systems), for IEEE JSTSP (Special Issue on Index Modulation for Future Wireless Networks: A Signal Processing Perspective), and for IEEE ACCESS (Special Section on Advances in Signal Processing for Non-Orthogonal Multiple Access). He has served on the Editorial Boards of several international journals, including the IEEE ACCESS, the EURASIP Journal on Wireless Communications and Networking, and the Physical Communication (Elsevier).

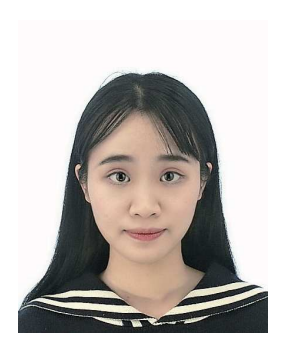

Xuan Chen received the B.S. degree from Wuhan University of Technology, Wuhan, China, in 2017. She is currently pursuing the Ph.D. degree with South China University of Technology, Guangzhou, China. Her main research interests include index modulation and molecular communications.

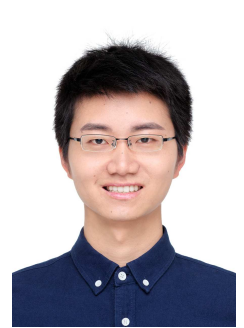

Qiang $\mathbf{L i}$ received the B.S. degree from Inner Mongolia University of Science and Technology, Baotou, China, in 2013, and the M.S. degree from Nanjing University of Aeronautics and Astronautics, Nanjing, China, in 2016. He is pursuing the Ph.D. degree with South China University of Technology, Guangzhou, China.

$\mathrm{He}$ is currently a Visiting Student Research Collaborator with Princeton University, Princeton, NJ, USA. His recent research interests include index modulation and non-orthogonal multiple access.

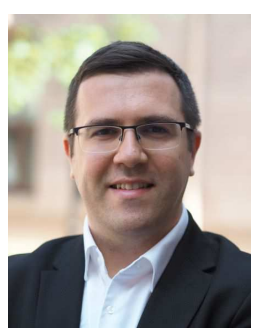

Ertugrul Basar (S'09-M'13-SM'16) received the B.S. degree (Hons.) from Istanbul University, Turkey, in 2007, and the M.S. and Ph.D. degrees from Istanbul Technical University, Turkey, in 2009 and 2013, respectively. He is currently an Associate Professor with the Department of Electrical and Electronics Engineering, Koç University, Istanbul, Turkey and the director of Communications Research and Innovation Laboratory (CoreLab). His primary research interests include MIMO systems, index modulation, waveform design, visible light communications, and signal processing for communications.

Recent recognition of his research includes the Science Academy (Turkey) Young Scientists (BAGEP) Award in 2018, Mustafa Parlar Foundation Research Encouragement Award in 2018, Turkish Academy of Sciences Outstanding Young Scientist (TUBA-GEBIP) Award in 2017, and the firstever IEEE Turkey Research Encouragement Award in 2017.

Dr. Basar currently serves as an Editor of the IEEE TRANSACTIONS on COMmunications and Physical Communication (Elsevier), and as an Associate Editor of the IEEE COMMUNICATIONS LETTERS. He served as an Associate Editor for the IEEE ACCESS from 2016 to 2018.

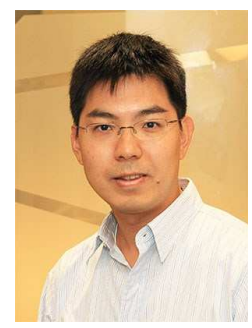

Yik-Chung Wu (S'99-M'05-SM'14) received the B.Eng. (EEE) degree in 1998 and the M.Phil. degree in 2001 from the University of Hong Kong (HKU). He received the Croucher Foundation scholarship in 2002 to study Ph.D. degree at Texas A\&M University, College Station, and graduated in 2005 From August 2005 to August 2006, he was with the Thomson Corporate Research, Princeton, NJ, as a Member of Technical Staff. Since September 2006, he has been with HKU, currently as an Associate Professor. He was a visiting scholar at Princeton University, in summers of 2015 and 2017. His research interests are in general areas of signal processing, machine learning and communication systems. Dr. Wu served as an Editor for IEEE COMMUNICATIONS LETTERS and IEEE TRANSACTIONS ON COMMUNICATIONS, and is currently an editor for JOURNAL OF COMMUNICATIONS AND NETWORKS.

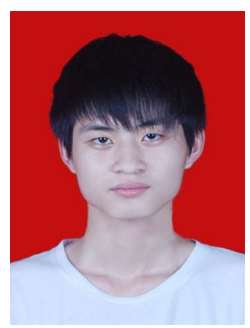

Wensong Zhang received the B.S. and M.S. degrees from the South China University of Technology, Guangzhou, China, in 2015 and 2018, respectively. His research interests include index modulation and OFDM. 